\title{
Tuning Concept and Design Criteria of Efficient Planar Metallic Plasmon Waveguides Using Nanocomposite Materials for Electromagnetic Radiation Applications
}

\author{
Alessandro Massaro ${ }^{1, *}$, Fabrizio Spano ${ }^{1}$, Roberto Cingolani ${ }^{2}$, and Athanassia Athanassiou ${ }^{1,3}$ \\ ${ }^{1}$ Center for Bio-Molecular Nanotechnologies (CBN) of IIT@UniLe, via Barsanti 1, 73010, Arnesano (LE), Italy \\ ${ }^{2}$ Italian Institute of Technology (IIT), Via Morego 30, 16163, Genova, Italy \\ ${ }^{3}$ National Nanotechnology Laboratory (NNL), CNR-Istituto di Nanoscienze, via per Arnesano, 73100 Lecce, Italy
}

\begin{abstract}
In this paper we propose a useful approach to enhance a surface plasmon wave (SPW) in a planar metallic waveguide obtained by nanocomposite materials. We prove analytically and experimentally that SPW propagates on the metallic surface and decays along the longitudinal direction of the plasmon waveguide. We develop a theoretical model based on the tuning of the effective permittivity index of a polymer film containing gold particles ranging from nano to micro scale. The tuning concept is based on the control of gold particles concentration in the polymeric film. The model starts from the accurate Brendel-Bormann (BB) method for the evaluation of the complex permittivity of metals and evaluates the cross section extinction and scattering by tuning the concentration of gold in a polymer film instead of metallic layer. Assuming the distance of gold nanoparticles (GN) small compared to the working wavelength, each particle behaves as an oscillator which is able to couple and transfer the SPW along the whole polymeric film layer as happens for thin metallic films. Under the hypothesis of uniform distribution of spherical GN in the nanocomposite material, the model shows the design criteria for high radiation efficiency of GN placed in the plasmon surface and coupled with the SPW. In order to highlight the importance of the dimensions of the GN concerning the light scattering process, we analyse the electromagnetic radiation pattern of GN characterised by different dimensions and excited by the resonant SPW obtained by the proposed model. The radiation pattern of spherical micro/nano particles is studied by means of a three dimensional (3D) finite element method (FEM). A good agreement between FEM and analytical results is found.
\end{abstract}

Keywords: Plasmon Waveguides, Tuning Approach, Gold Micro Nano Particles, Radiation Efficiency, Nanocomposite Design.

\section{INTRODUCTION}

The plasmon is a quantum of plasma oscillation, i.e., a quasiparticle resulting from the quantization of plasma oscillations just like photons and phonons are quanta of light and sound waves, respectively. Thus, plasmons are collective oscillations of the free electron gas density at specific frequencies. Surface Plasmon waves (SPW) are those plasmons that are confined to surfaces and strongly interact with light. They occur at the interface between air or a material with a positive dielectric constant and a material with a negative dielectric constant (usually a metal or a doped dielectric). The optical properties of metallic nanostructures have recently been the

${ }^{*}$ Author to whom correspondence should be addressed. focus of intense study, because upon interaction with electromagnetic waves, the metal nanostructures exhibit localized surface plasmon resonances. ${ }^{1-3}$ Surface plasmons can be thought of as light waves trapped at the surfaces of the metal due to interactions with the conduction electrons of the metal. In response, the conduction electrons of the metal may oscillate in resonance with the light waves at specific incident frequencies, which depend on the metal properties. The specific wavelength at which the resonance occurs is known as the plasmon resonance, which manifests itself through light emission. Metal nanostructures that strongly absorb light are technologically interesting, since the light absorbed by the nanostructure can be locally released as heat. Therefore, researchers have utilized strongly absorbing metal nanostructures for a variety of applications, such as localized 
photothermal ablation treatment of cancer. ${ }^{4-6}$ Conversely, the ability of the metal nanostructures to strongly scatter light is critical for optical sensing, tagging and imaging applications. ${ }^{7,8}$ Applications involving light scattering are numerous, and include the use of metal nanostructures as contrast agents in cancer cell imaging, ${ }^{9,10}$ the use of composite metal nanostructures for optical labeling and tagging applications, ${ }^{11,13}$ and the use of metal nanostructures as optically active strain sensors. ${ }^{14}$ Furthermore, it is important to note that the relative contributions of absorption and scattering to the overall extinction spectra (exctinction $=$ scattering + absorption) is a function of the nanoparticle size; for example, Jain et al. ${ }^{15}$ showed, by classical electrodynamic calculations, that the extinction spectra of small gold nanoparticles (GN), with diameters less than about $50 \mathrm{~nm}$, is dominated by absorption, whereas scattering becomes increasingly important for larger diameters. Since the specific optical properties of the metal nanostructures, i.e., absorption, scattering and local electric field, are sizedependent, the size control of the GN somehow dictates the final application. In this frame it is of considerable interest to investigate methods by which the radiation field spectra and local electric field can be enhanced, and to study the enhancement as a function of the nanostructure size. The proposed work is based on a new concept of plasmon wave enhancement and introduces the plasmonic field enhancement as a function of the tuning of metal concentration in a matrix, which replaces the metallic layer of a planar plasmonic waveguide. Starting from the BrendelBormann (BB) method ${ }^{16}$ which describes the experimental data $^{16}$ about the complex permittivity of metals in a wide spectral range, our analytical approach extends the model to the design of an efficient plasmon waveguide constituted by spherical GN of different concentration in a polymer. In particular the paper is organized as follows:

(1) We develop an accurate theoretical model of planar metallic plasmon waveguide including evaluation of SPW propagation constants, of permittivity, and losses.

(2) We validate the plasmon wave theory by comparing experimental results with analytical ones.

(3) We design an efficient plasmon waveguide by calculating the extinction and scattering efficiency.

(4) Finally, we compare the radiation pattern between 3D FEM and analytical results of spherical gold micro/nano particles placed on the designed waveguide.

\section{THEORETICAL MODEL: PROPAGATION IN PLASMON WAVEGUIDES AND PERMITTIVITY EVALUATION OF THE METALLIC LAYER}

We define in this section the theoretical model of the planar metal plasmon waveguide depicted in Figure 1.

Assuming as source an electric field $E_{0}$ parallel polarized to the plane of incidence (according with the $p$-polarized light which is able to excite electronic surface plasmons), the resulting surface electromagnetic wave will have the following general form

$$
\begin{aligned}
& \mathbf{A}_{1}=\mathbf{A}_{\mathbf{1 0}} e^{i\left(\mathbf{k}_{\mathbf{x} 1} \mathbf{x}+\mathbf{k}_{z, \mathrm{EW}} \mathbf{z}-\omega t\right)} \quad x<0 \\
& \mathbf{A}_{2}=\mathbf{A}_{20} e^{i\left(\mathbf{k}_{\mathbf{x} 2} \mathbf{x}+\mathbf{k}_{z, \text { SPW }} \mathbf{z}-\omega t\right)} \quad x>0
\end{aligned}
$$

where $\mathbf{A}$ stands for $\mathbf{E}$ and $\mathbf{H}, \mathbf{k}_{\mathbf{x} 1}$ and $\mathbf{k}_{\mathbf{x} 2}$ are the wavevectors in the $x$-direction, $\mathbf{k}_{z \text {, EW }}$ and $\mathbf{k}_{z, \mathrm{SPW}}$ are those in the $z$-direction and $\omega$ is the angular frequency. Both fields $\mathbf{E}$ and $\mathbf{H}$ must fulfill the Maxwell equations:

$$
\begin{gathered}
\nabla \cdot \mathbf{H}=0 \\
\nabla \cdot \mathbf{E}=0 \\
\nabla \times \mathbf{E}+\frac{1}{c} \frac{\partial \mathbf{H}}{\partial t}=0 \\
\nabla \times \mathrm{H}+\frac{\varepsilon}{c} \frac{\partial \mathbf{E}}{\partial t}=0
\end{gathered}
$$

The incident light coming from the dielectric material reflects at the metallic interface inducing light localization, called evanescent field (EW). The EW excites the SPW which propagates along the surface of the metal film and thus, a part of the incident light is absorbed. The light intensity of the reflected ray depends on the incidence angle $\theta$. The propagation constant of the EW is expressed as

$$
k_{z, \mathrm{EW}}=\frac{2 \pi}{\lambda_{0}} \sqrt{\varepsilon_{c}} \sin \theta
$$

where $\lambda_{0}$ is the working wavelength. The propagation constant of the SPW wave is given by the following expression $^{16,17}$

$$
\begin{gathered}
k_{d}=\frac{2 \pi}{\lambda_{0}}\left(\frac{\varepsilon_{a} \varepsilon_{m}}{\varepsilon_{a}+\varepsilon_{m}}\right)^{1 / 2} \\
k_{r}=-k_{d}\left(\frac{2}{\varepsilon_{a}-\varepsilon_{m}}\right)\left(\frac{\varepsilon_{a} \varepsilon_{m}}{\varepsilon_{a}+\varepsilon_{m}}\right)^{3 / 2} \\
\times \exp \left\{i \frac{4 \pi d}{\lambda_{0}} \frac{\varepsilon_{m}}{\left(\varepsilon_{a}+\varepsilon_{m}\right)^{1 / 2}}\right\}
\end{gathered}
$$

where $k_{d}$ is the dispersion relation of the SPW of a metalair interface in a one-sharp-boundary model, $k_{r}$ is the perturbation to $k_{d}$ due to the physical volume of the metal defined by the thickness $d$.

When Eqs. (3) and (4) satisfy the following equation, the surface plasmon resonance occurs and a part of incident light is absorbed:

$$
k_{z, \mathrm{SPW}}=k_{d}+k_{r}
$$

The complex dielectric function of a metallic film can be expressed in the following form:

$$
\varepsilon_{m}(\omega)=\varepsilon_{m}^{\prime}(\omega)+i \varepsilon_{m}^{\prime \prime}(\omega)
$$




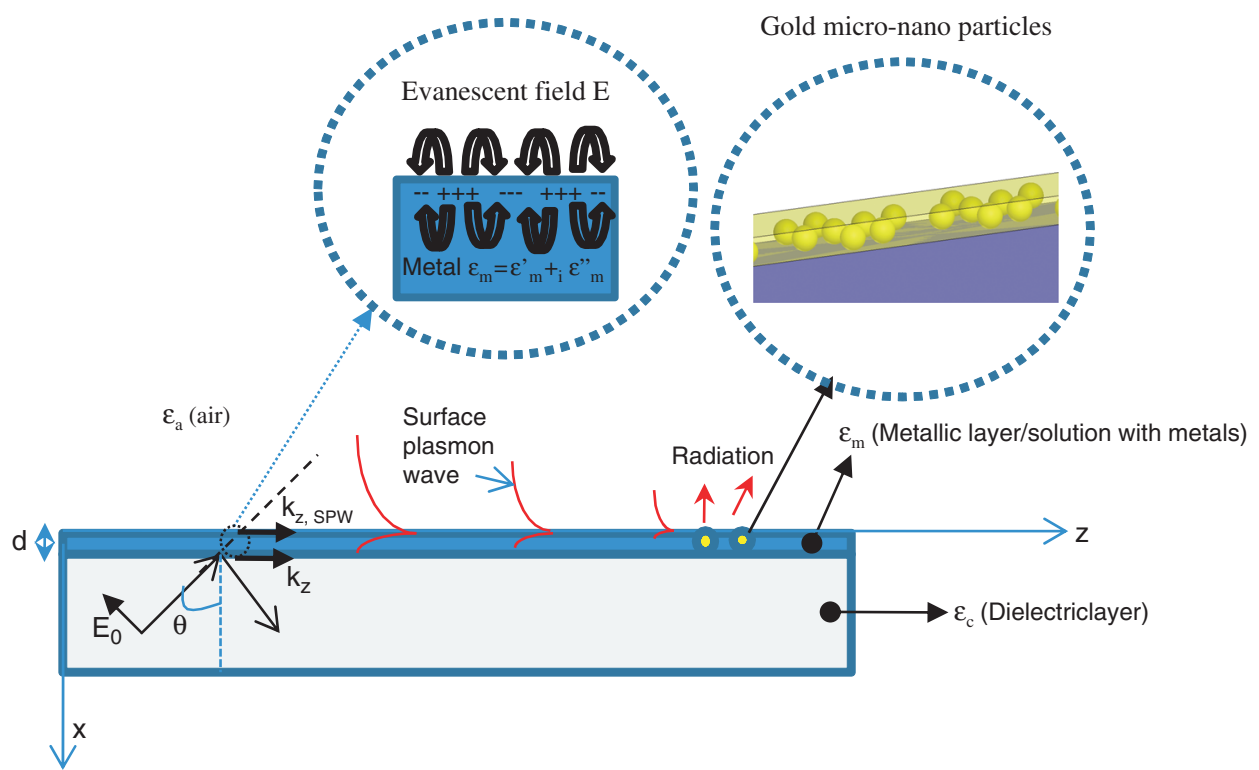

Fig. 1. Plasmon waveguide and evanescent field $\mathbf{E}$ (surface plasmon waves) propagating along the $z$-direction and impinging on gold micro-nano particles. The radiation effect of gold micro-nano particles can be enhanced by tuning the gold concentration of the polymeric film.

According with the Brendel-Bormann (BB) model the expression (6) becomes ${ }^{16}$

$$
\varepsilon_{m}(\omega)=1-\frac{\Omega_{p}^{2}}{\omega\left(\omega-i \Gamma_{0}\right)}+\sum_{j=1}^{k} \chi_{j}(\omega)
$$

where $k$ is the number of $\mathrm{BB}$ oscillators used to interpret the intraband part of the spectrum, $\Omega_{\mathrm{P}}=\left(f_{0} \omega_{\mathrm{P}}\right)^{1 / 2}$ is the plasma frequency associated with intraband transition with oscillators strength $f_{0}$ and damping constant $\Gamma_{0}$, and with the infinite number of oscillators (part which replaces the Lorentz oscillators) given by ${ }^{16}$

$$
\begin{aligned}
\chi_{j}(\omega)= & \frac{1}{\sqrt{2 \pi} \sigma_{j}} \int_{-\infty}^{\infty} \exp \left[\frac{-\left(t-\omega_{j}\right)^{2}}{2 \sigma_{j}^{2}}\right] \\
& \times \frac{f_{j} \omega_{p}^{2}}{\left(t^{2}-\omega^{2}\right)+i \omega \Gamma_{j}} d t
\end{aligned}
$$

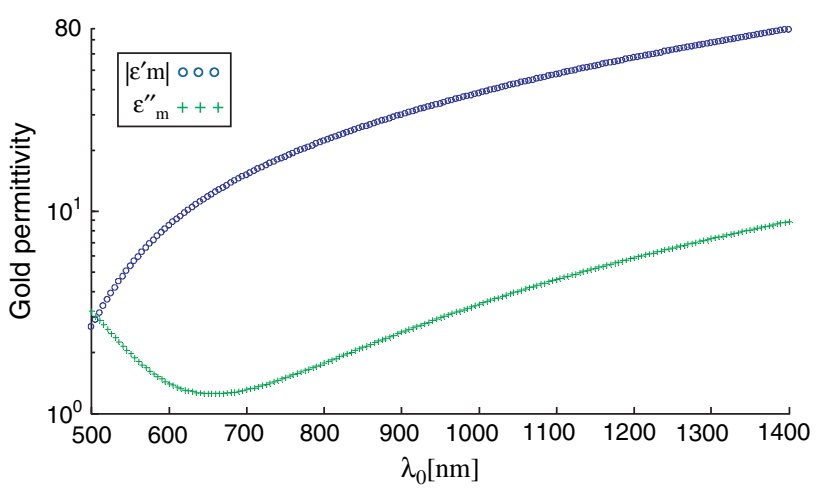

Fig. 2. Gold permittivity versus the wavelength calculated by the BB model. The parameters of Eq. (7) are defined in Ref. [15].
In Figure 2 is reported the calculation of gold complex permittivity by the BB approach, and in Figure 3 is illustrated the electric field distribution for a planar waveguide with gold metallic layer where the complex permittivity is evaluated by (6) and (7). The propagation constants $(5) k_{z, \mathrm{SPW}}=k_{z \text {, SPW }}^{\prime}+i k_{z, \mathrm{SPW}}^{\prime \prime}$ of the SPW define the electromagnetic field profile and change with the working wavelength and with the thickness $d$. In Figure 4 and in Figure 5 are reported the real part and the imaginary part of the propagation constant $k_{z, \mathrm{SPW}}$, respectively, versus the working wavelength for different gold layer thickness $d$. We note that the evanescent propagating field

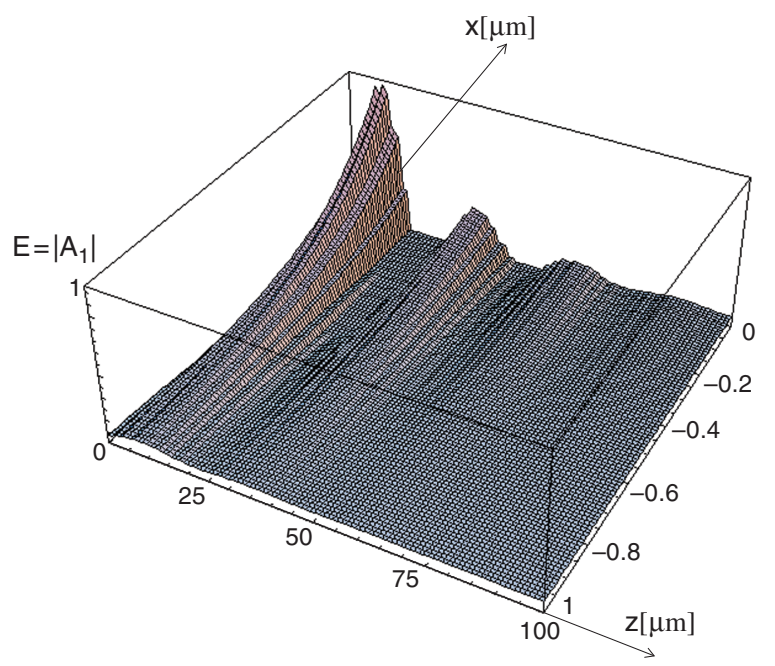

Fig. 3. Evanescent field distribution in air region $(x<0)$ for a particular time-step. The waveguide is characterised by a gold metallic layer at $x=0$ with $d=50 \mathrm{~nm}$. The working wavelength is $\lambda_{0}=1.55 \mu \mathrm{m}$, and $\varepsilon_{c}=3.1, \varepsilon_{a}=1$. 


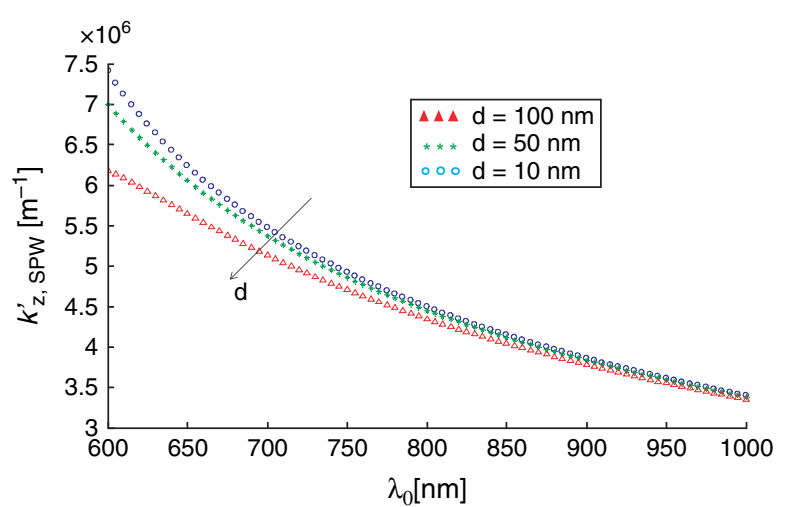

Fig. 4. Real part of the propagation constant $k_{z \text {, SPW }}$ versus the working wavelength $\lambda_{0}$ for different values of gold layer thickness.

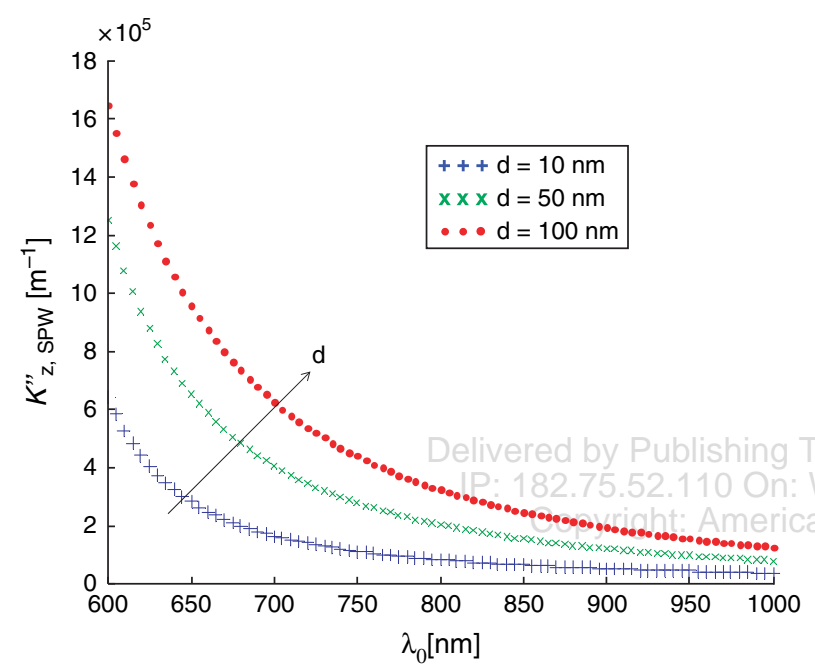

Fig. 5. Imaginary part of the propagation constant $k_{z, \text { SPW }}$ versus the working wavelength $\lambda_{0}$ for different values of gold layer thickness.

is $E \sim e^{z \cdot k^{\prime \prime} z \text {, SPW }} \cdot e^{-i z \cdot k^{\prime} z \text {, SPW }}$, consequently the energy lost along the $z$-propagation direction will be estimated by $k_{z \text {, SPW }}^{\prime \prime}$ which increases with the thickness $d$ (see Fig. 5).

\section{TESTING THE THEORETICAL MODEL OF SPW PROPAGATION: COMPARISON WITH EXPERIMENTAL RESULTS}

For a simple validation of the theoretical modeling, we measured the evanescent field intensity of a waveguide with indium tin oxide (ITO $)^{19,20}$ as top layer. The plasmon waveguide with metallic ITO layer is an excellent model system for the study of surface plasmons ${ }^{19}$ because the material properties can be tuned systematically by variation of the preparation conditions. In particular by considering the experimental setup of Figure 6 it is possible to quantify the plasmonic evanescent field intensity by attaching an optical fiber as detector directly on the ITO surface. A light probe beam (from a tungsten broad band lamp) is injected into the plasmonic waveguide through

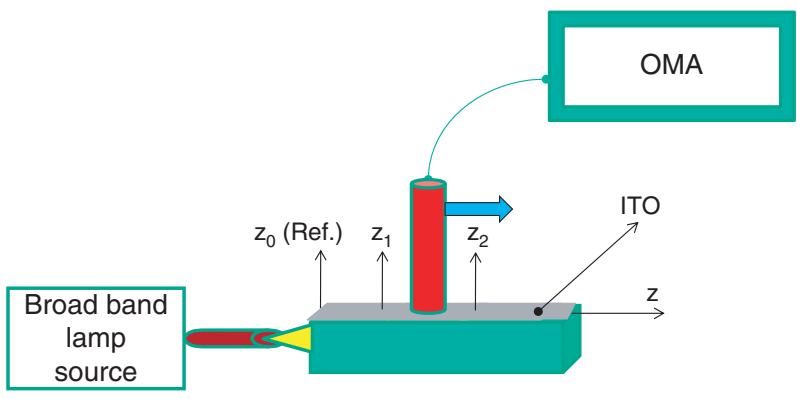

Fig. 6. Experimental setup for evanescent field detection.

a tapered fiber which is not attached to the waveguide in order to improve a plane wave source. The light exciting the waveguide and radiated by the metallic ITOlayer is collected and collimated by a multimode fiber positioned directly above the ITO layer connected to a $\mathrm{N}$-cooled InGaAs OMA (optical multichannel analyzer). We measure the evanescent field of the ITO waveguide characterized by $d=100 \mathrm{~nm}, \varepsilon_{c}$ (glass) $=5.5$ by shifting the attached fiber/detector along the $z$-longitudinal direction. The power transferred by the waveguide decreases with the distance $z$. In particular for a particular $z$-position of the fiber of Figure 6 we estimate the following normalized quantity:

$$
P(z)=\left|A_{1}(\omega, z) / A_{1}\left(\omega, z_{0}\right)\right|^{2}
$$

where $z_{0}$ represents the reference $z$-position indicating the waveguide input. The comparison between experimental

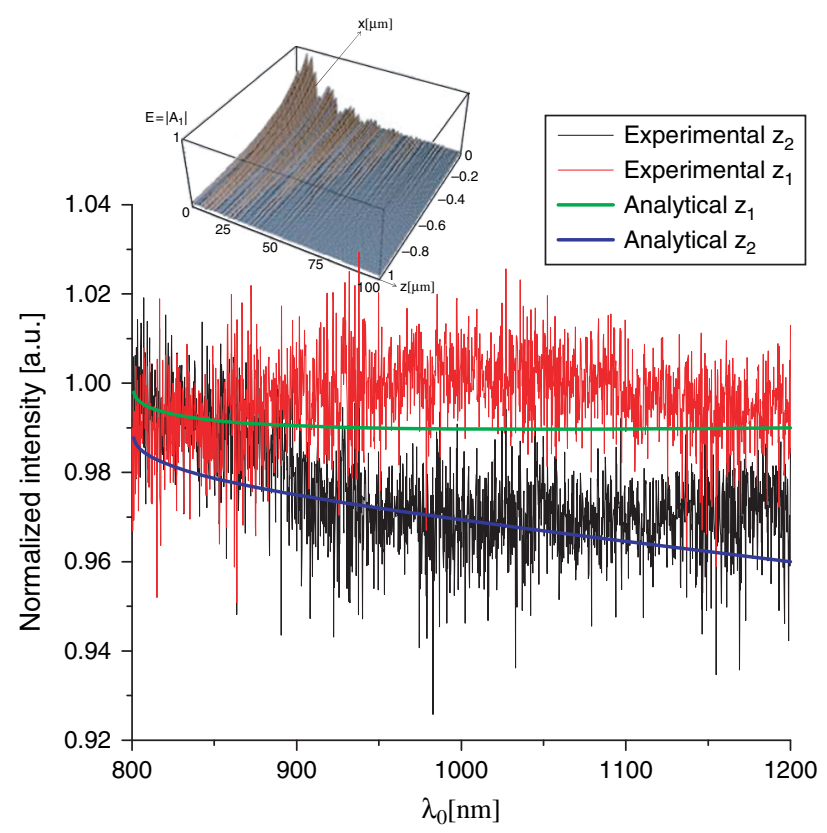

Fig. 7. Comparison between experimental and analytical results of the evanescent field intensity for an ITO plasmon waveguide. The intensity is normalized with respect to the intensity measured/calculated at $z=z_{0}$. Inset: evanescent field distribution related to in air region $(x<0)$ for a particular time-step. The value of $\varepsilon_{m}$ used in the calculus are reported in Ref. [19]. 
and analytical evanescent field intensity is reported in Figure 7. We observe from Figure 7 that at the position $z_{2}$ the evanescent field decreases by following the analytical trend. A good agreement between experimental and analytical results is found.

\section{DESIGN CRITERIA: CONCENTRATION TUNING OF GOLD PARTICLES AND RADIATION ASPECTS}

As discussed before the intensity of SPW decays along the longitudinal $z$-direction. For this purpose, an optimization of the waveguide operating under plasmon resonance condition is important, especially for applications where the coupling between waveguide and nano metallic particles is required. For this purpose we study in this section the possibility to enhance the SPW by tuning the concentration of the metal in a polymer film.

The effective dielectric function $\varepsilon_{\text {eff }}$ for spherical metallic particles having dielectric function $\varepsilon_{m}$ embedded in a medium (polymer film) $\varepsilon_{s}$ is defined as ${ }^{21}$

$$
\varepsilon_{\mathrm{eff}}=\varepsilon_{s} \frac{\varepsilon_{m}(1+2 \phi)+2 \varepsilon_{s}(1-\phi)}{\varepsilon_{m}(1-\phi)+\varepsilon_{s}(2+\phi)}
$$

where $\phi=0 \ldots 1$ indicates the metal concentration as volume filling factor. As illustrated in Figure 8, the effective dielectric permittivity allows us to approximate a polymeric film with a defined concentration of metallic micro-nano particles (characterized by different dimensions) with a metallic equivalent film. By assuming metallic GN with uniform diameter the equivalent metallic layer can be approximated with a thin metallic film with thickness equal to the GN diameter. This equivalence is possible because we suppose that the particles are placed in the

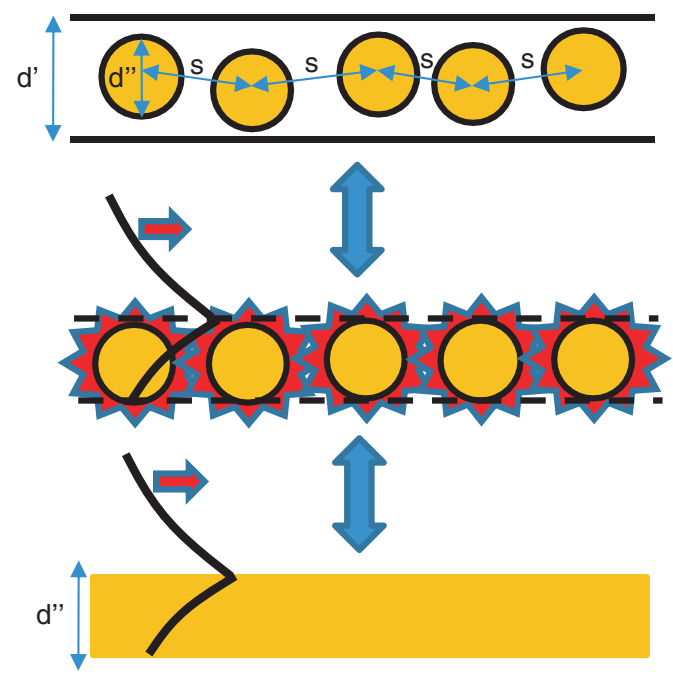

Fig. 8. Effective permittivity approach which allows to approximate a polymeric film with gold particles as a metallic thin film. The thickness $d^{\prime \prime}$ is a function of the diameter of the gold nanoparticles if the various distances $s$ are $\leq \lambda_{o}$.

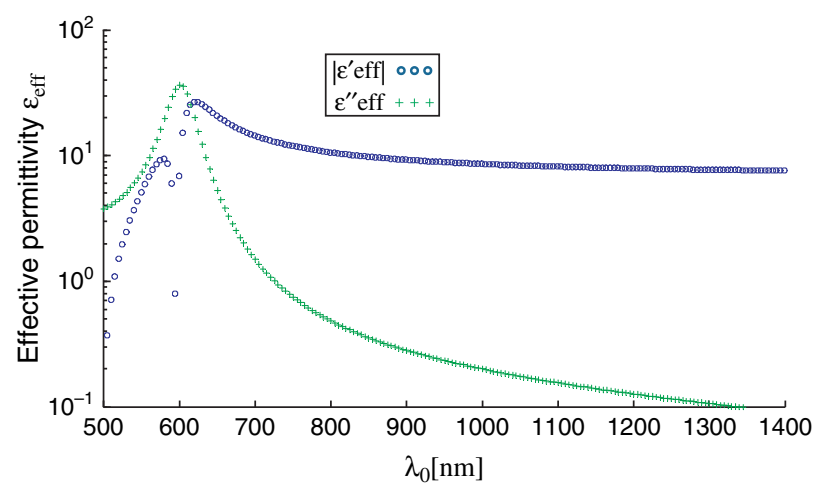

Fig. 9. Total effective dielectric function of spherical gold micro-nano particles in polymer film.

polymeric film at various distances $s$ small compared to the working wavelength: in this case each particle behaves as a plasmonic oscillator and allows the propagation of the SPW along the whole surface. If we consider small distances $s$ and GN with different diameters will be possible in any case to calculate the effective permittivity by the filling factor $\phi$. GN with different dimensions introduce a further complexity regarding the modelling of the light scattering characterization (random scattering).

The polymer of choice was PDMS since it is suitable for the direct generation of GN in the polymeric matrix in the presence of suitable precursors. ${ }^{22,23}$ The effective dielectric function of PDMS polymer film with gold micro/nano particles having the same radius and with $\phi=0.5$ is illustrated in Figure 9. Comparing Figure 2 with Figure 9 we observe a strong variation of the complex permittivity and, consecutively, the resonance condition will change.

When a small spherical metallic micro/nano particle is irradiated by light, the oscillating electric field causes the conduction electrons to oscillate coherently. This is schematically pictured in Figure 10. The electric field $\mathbf{E}_{\text {out }}$ outside the single sphere can be approximated for small particles (dipole Plasmon resonance) to ${ }^{24}$

$$
\mathbf{E}_{\text {out }}=\mathbf{A}_{1}-g_{d} A_{1}\left[\frac{\mathbf{x}}{r^{3}}-\frac{3 x}{r^{5}}(x \mathbf{x}+y \mathbf{y}+z \mathbf{z})\right]
$$

where $\mathbf{x}, \mathbf{y}$ and $\mathbf{z}$ are the usual unit vectors, $\mathbf{A}_{1}$ is the SPW calculated by (1), $g_{d}$ is the normalized sphere polarizability

$$
g_{d}=\frac{\varepsilon_{m}-\varepsilon_{\text {eff }}}{\varepsilon_{m}+2 \varepsilon_{\text {eff }}}
$$

being $a$ the radius of the sphere. The resonance conditions can be estimated by calculating the extinction efficiency

$$
Q_{\text {dip.ext. }}=4 u \operatorname{Im}\left(g_{d}\right)
$$

with $u=2 \pi\left(\varepsilon_{\text {eff }}\right)^{1 / 2} / \lambda_{0}$. In order to estimate the scattering contribution to the efficiency we consider also the following scattering efficiency

$$
Q_{\text {dip.scat. }}=\frac{8}{3} u^{4}\left|g_{d}\right|^{2}
$$




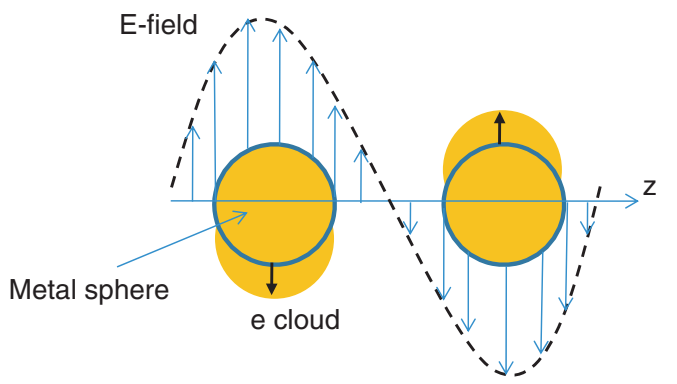

Fig. 10. Schematic of plasmon oscillation for a sphere, showing the displacement of the conduction electron charge cloud relative to the nuclei.

For larger particles (quadrupole plasmon resonance) in microscale, the resulting field outside the sphere becomes

$$
\begin{aligned}
\mathbf{E}_{\text {out }}= & \mathbf{A}_{1}+i \frac{2 \pi}{\lambda_{0}} A_{1}[x \mathbf{x}+z \mathbf{z}]-g_{d} A_{1} \\
& \times\left[\frac{\mathbf{x}}{r^{3}}-\frac{3 x}{r^{5}}(x \mathbf{x}+y \mathbf{y}+z \mathbf{z})\right] \\
& -g_{q} A_{1}\left[\frac{x \mathbf{x}+z \mathbf{z}}{r^{5}}-\frac{5 z}{r^{7}}(x \mathbf{x}+y \mathbf{y}+z \mathbf{z})\right]
\end{aligned}
$$

with the normalized quadrupole polarizability $g_{q}$ given by

$$
g_{q}=\frac{\varepsilon_{m}-\varepsilon_{\text {effere }}}{\varepsilon_{m}+3 / 2 \varepsilon_{\text {eff }}}
$$

The dipole + quadrupole extinction and Rayleigh scattering efficiency will be defined as

$$
\begin{aligned}
Q_{\text {quad.ext. }} & =4 u \operatorname{Im}\left[g_{d}+\frac{u^{2}}{12} g_{q}+\frac{u^{2}}{30}\left(\varepsilon_{m}-1\right)\right] \\
Q_{\text {quad.ext }} & =\frac{8}{3} u^{4}\left\{\left|g_{d}\right|^{2}+\frac{u^{2}}{12}\left|g_{q}\right|^{2}+\frac{u^{4}}{900}\left|\varepsilon_{m}-1\right|^{2}\right\}
\end{aligned}
$$

We observe that for a couple of small nano particles the resulting field of the system $\mathbf{E}_{\text {out } 2}$ can be calculated by

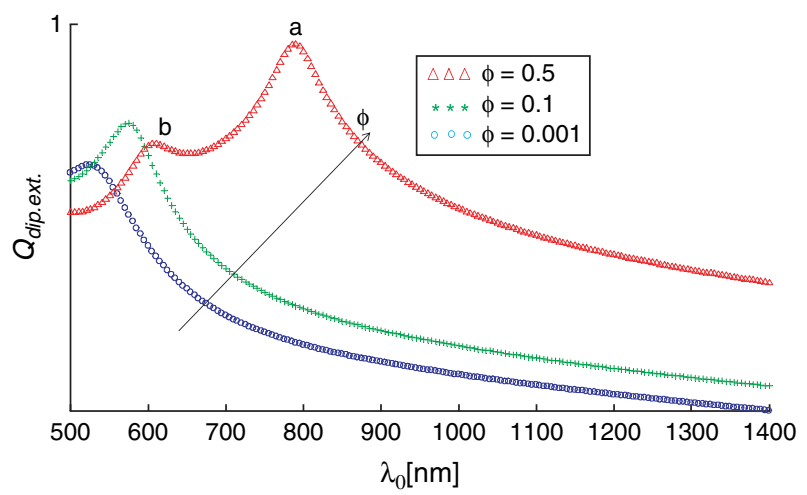

Fig. 11. Dipole extinction efficiency for different gold concentrations $\phi$.

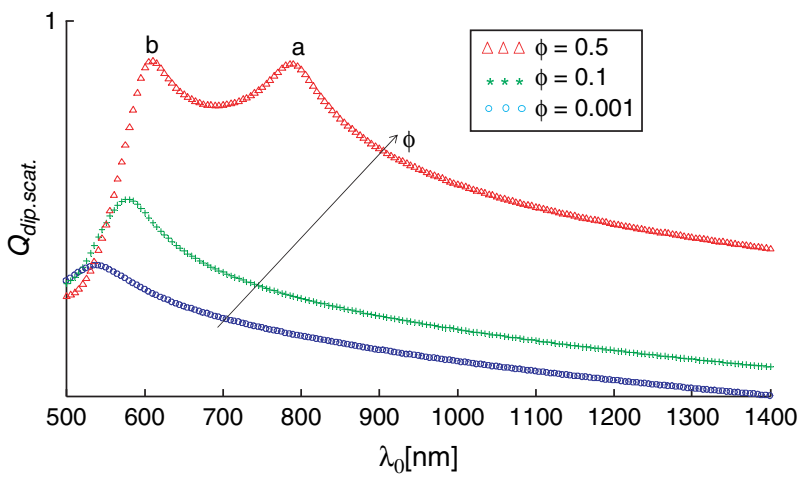

Fig. 12. Dipole scattering efficiency for different gold concentrations $\phi$.

applying twice Eq. (11) as indicated by the following approximation related to a couple of particles

$$
\begin{aligned}
& \mathbf{E}_{\text {out } 1}=\mathbf{A}_{1}-g_{d 1} A_{1}\left[\frac{\mathbf{x}}{r^{3}}-\frac{3 x}{r^{5}}(x \mathbf{x}+y \mathbf{y}+z \mathbf{z})\right] \\
& \mathbf{E}_{\text {out } 2}=\mathbf{E}_{\text {out } 1}-g_{d 2} \mathbf{E}_{\text {out } 1}\left[\frac{\mathbf{x}}{r^{3}}-\frac{3 x}{r^{5}}(x \mathbf{x}+y \mathbf{y}+z \mathbf{z})\right]
\end{aligned}
$$

Assuming $a=60 \mathrm{~nm}$ (spherical GN in PDMS polymeric film) we calculate in Figure 11 the dipole extinction efficiency which provides the plasmon resonances. A major resonance effect is observed by increasing the gold concentration $\phi$; for $\phi=0.5$ we found two working wavelengths corresponding to the points a and $\mathrm{b}$ of Figure 11: this is due to the fact that as the concentration increases, scattering becomes the dominant contribution to the nano particle extinction, as proved by the scattering efficiency of Figure 12. Figure 13 shows that by increasing the size of the gold particles, the working wavelength around $800 \mathrm{~nm}$ found for small GN $(60 \mathrm{~nm})$ shifts towards shorter wavelengths. The calculated power $\left|\mathbf{E}_{\text {out }}\right|^{2}$ for a single spherical GN reported in Figure 14 establishes that other spherical gold particles can be coupled in a $y$-z-spatial range between 0 and $1 \mu \mathrm{m}$.

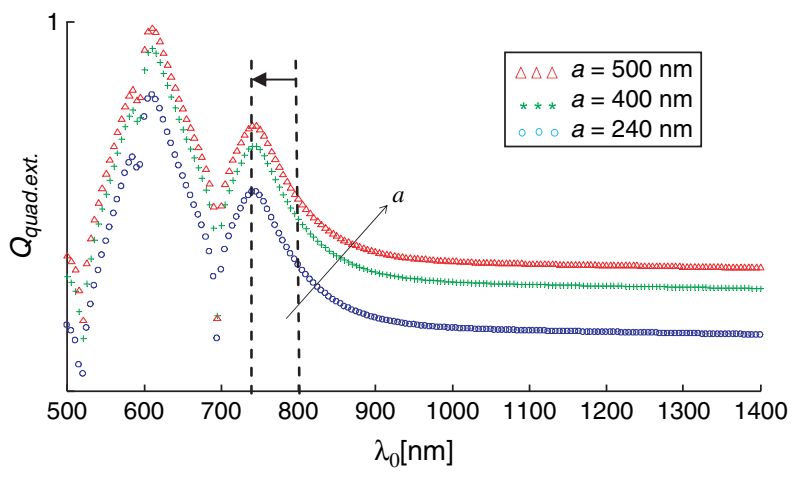

Fig. 13. Quadrupole extinction efficiency for different gold radius $a$. $\phi$ is fixed to 0.5 . Dashed lines indicate the displacement of the wavelength due to the variation of nanoparticle size if compared with the plots of Figures 11 and 12 where are reported the extinction of particles with a size of $60 \mathrm{~nm}$. 


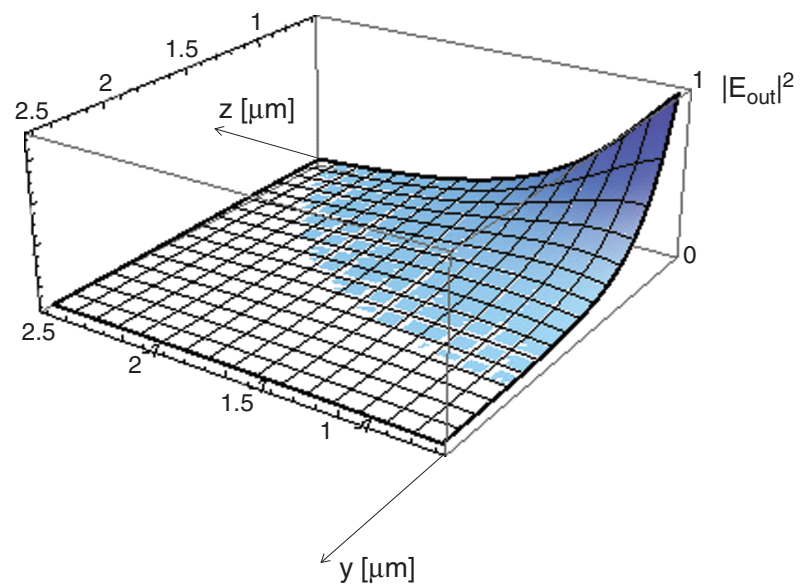

Fig. 14. Normalised power outside a spherical gold particle with $a=$ $60 \mathrm{~nm}$ in polymeric film. The plot indicates the coupling efficiency versus the distances $s$ between two nanoparticles (see Fig. 8).

\section{UNIFORM SCATTERING AND COMPARISON WITH 3D FEM APPROACH}

In order to validate the theoretical model we compare the results with a properly designed 3D FEM tool typically used for the design of micrometric structures. ${ }^{25}$ By assuming spherical gold particles spaced by a distance $s$ (see Fig. 8) low compared to the working wavelength, we characterized the gold GN by means of the radiated field. This characterization is useful in order to study the electromagnetic behaviour of micro-nano probes by means of near field detection. The FEM near field region is the region closest to the source (parallel-plate waveguide with aperture), and the electric field $\mathbf{E}(x, y, z)$ external to the region bounded by a closed spherical surface may be written as ${ }^{26}$

$$
\begin{aligned}
\mathbf{E}(x, y, z) & =\int_{S}\left(\left(j \omega \mu_{0} \mathbf{H}_{\tan }\right) G+\left(\mathbf{E}_{\tan } \times \nabla G\right)\right. \\
& \left.+\left(\mathbf{E}_{\text {normal }} \nabla G\right)\right) d S
\end{aligned}
$$

where $S$ represents the radiation boundary surface, $j$ is the imaginary unit, $\mathbf{H}_{\tan }$ is the component of the magnetic field that is tangential to the surface, $\mathbf{E}_{\text {normal }}$ is the component of the electric field that is normal to the surface, $G$ is the free space Green's function, given by

$$
G=\frac{e^{-i k\left|\mathbf{r}-\mathbf{r}^{\prime}\right|}}{\left|\mathbf{r}-\mathbf{r}^{\prime}\right|}
$$

(a)

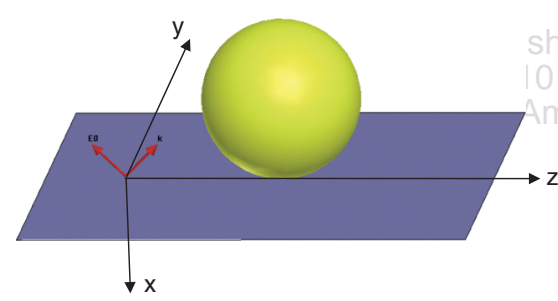

(b)

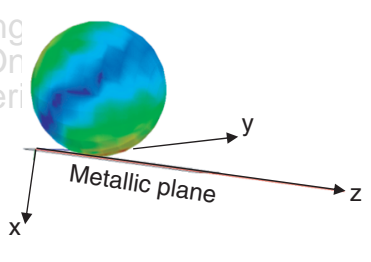

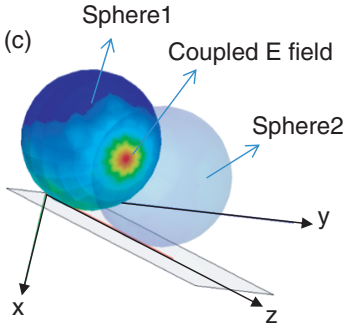

Fig. 15. (a) 3D FEM modeling of spherical gold nanoparticle on a metallic plane: the excitation $\mathbf{E}_{\mathbf{0}}=\mathbf{A}_{\mathbf{1}}$ is oriented as illustrated in Figure 1. (b) electric field coupled on the surface of a single gold nanoparticle. (c) Coupling between two attached gold nanoparticles.

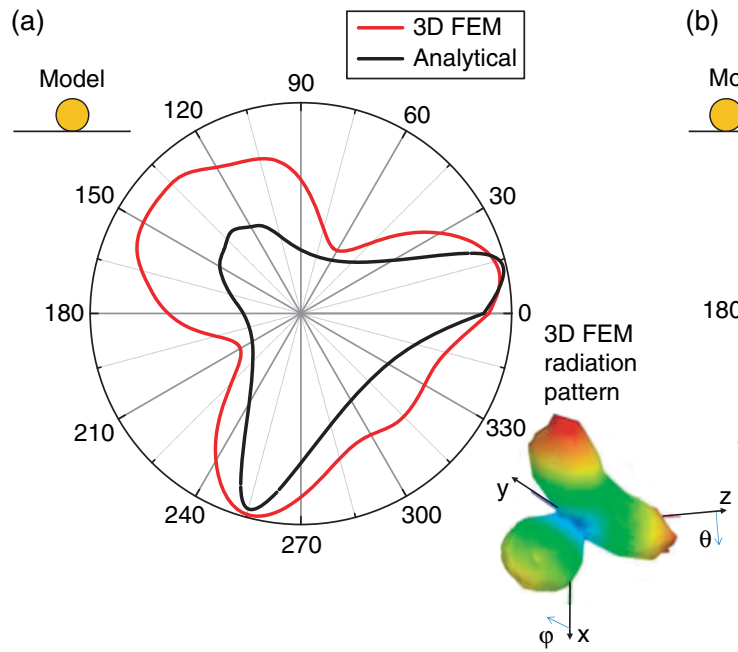

(a)

(b)

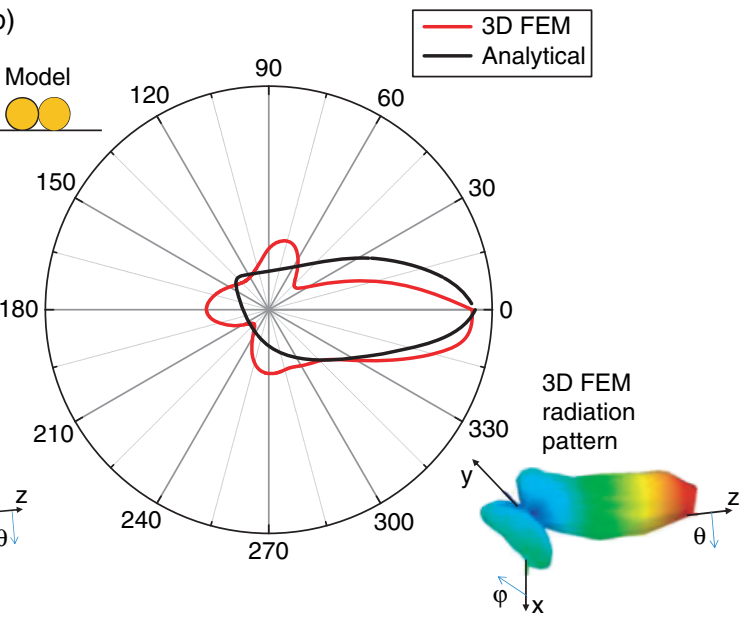

Fig. 16. (a) Comparison between analytical and 3D FEM radiation pattern versus $\theta$ at $\varphi=0$ of a single spherical nanoparticle excited by a SPW at resonance wavelength $\lambda_{0}=800 \mathrm{~nm}$. Inset (above): 3D fem modelling. Inset (below): 3D FEM radiation pattern. (b) Comparison between analytical and 3D FEM radiation pattern versus $\theta$ at $\varphi=0$ of a coupled attached spherical nanoparticle excited by a SPW at resonance wavelength $\lambda_{0}=800 \mathrm{~nm}$. Inset (above): 3D FEM modelling. Inset (below): 3D FEM radiation pattern. 


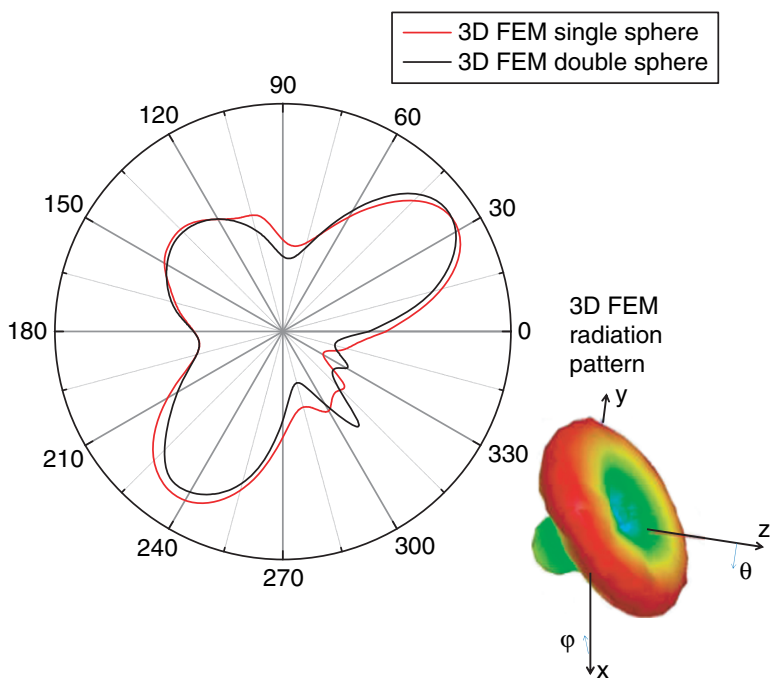

Fig. 17. 3D FEM radiation pattern versus $\theta$ at $\varphi=0$ of a single and of an attached couple of spherical micro-particle excited by a SPW at resonance wavelength $\lambda_{0}=800 \mathrm{~nm}$. The diameter of the spherical gold particles is $2 a=1 \mu \mathrm{m}$. Inset (below): 3D FEM radiation pattern.

being $k$ the free space wave number, and, $\mathbf{r}$ and $\mathbf{r}^{\prime}$ representing field points and source points on the surface, respectively. Figure 14(a) illustrated the 3D FEM modelling of a gold spherical nanoparticle with $a=$ $60 \mathrm{~nm}$ placed in the equivalent metallic surface (thin film ground plane boundary condition): the nanoparticle is excited at the resonance wavelength (see Fig. 11 point a) $\lambda_{0}=800 \mathrm{~nm}$ with a $p$-polarised electric field $\mathbf{E}_{0}=\mathbf{A}_{1}$. Figures 15(b) and (c) show the 3D electric field distribution on the spherical surface for a single sphere and for two spheres, respectively. For nanoshell configurations, we consider in Figure 15(c) two attached equal spheres (uniform scattering problem) to determine the maximum coupling effect. Figures 16(a) and (b) show the good comparison agreement between analytical and 3D FEM radiation pattern for a single spherical nanoparticle and the couple of nanoparticles, respectively. We note that the radiation pattern of the couple of spheres has the maximum lobe only along the $z$-direction by defining a preferred direction of the radiation which is potentially useful for a radiation sensors. In the hypothesis that the nanoparticles cluster into micro-agglomerates at $\lambda_{0}=800 \mathrm{~nm}$, the radiation pattern of Figure 16 will change in Figure 17: we observe from Figure 17 that by increasing the dimension of the particles (even by agglomeration), the radiation pattern will converge in a well defined symmetric lobes configuration by controlling better the near field (aspect useful especially for biosensing applications).

\section{CONCLUSION}

We present in this work the theoretical model of efficient planar metallic plasmon waveguides. The analysis of the waveguide allows us to characterise the radiation efficiency of spherical micro-nano particles embedded in a polymeric film by tuning the concentration of the metal playing with the gold concentration. The study is extended also to a couple of spherical micro-nano particles placed on a resonant waveguide. The comparison with experimental results and 3D FEM models proves that the proposed model is a valid tool for the design of nanocomposite materials for specific applications to micro-nano antennas.

The tuning concept and the radiation approach can be extended also to a complex controlled nanoshell configuration. The approach will be applied for the interpretation of future experimentations concerning the evanescent field generated by gold nanocomposite polymeric films for electromagnetic field enhancement applications.

\section{References}

1. E. Ozbay, Science 311, 189 (2006).

2. J. R. Sambles, G. W. Bradbery, and F. Yang, Contemp. Phys. 32, 173 (1991).

3. W. L. Barnes, A. Dereux, and T. W. Ebbeso, Nature 424, 824 (2003).

4. L. R. Hirsch, A. M. Gobin, A. R. Lowery, F. Tam, R. A. Drezek, N. J. Halas, and J. L. West, Ann. Biomed. Eng. 34, 15 (2006).

5. L. R. Hirsch, R. J. Stafford, J. A. Bankson, S. R. Sershen, B. Rivera, R. E. Price, J. D. Hazle, N. J. Halas, and J. L. West, Proceedings of the National Academy of Science 100, 13549 (2003).

6. X. Huang, I. H. El-Sayed, W. Qian, and M. A. El-Sayed, Journ. Am. Chem. Soc. 128, 2115 (2006).

7. G. Raschke, S. Brogl, A. S. Susha, A. L. Rogach, T. A. Klar, J. Feldmann, B. Fieres, N. Petkov, T. Bein, A. Nicht, and K. Kurzinger, Nano Lett. 4, 1853 (2004).

8. M. D. Malinsky, K. L. Kelly, G. C. Schatz, and R. P. V. Duyne, Journ. Am. Chem. Soc. 123, 1471 (2001).

9. K. Sokolov, M. Follen, J. Aaron, I. Pavlova, A. Malpica, R. Lotan, and R. Richards-Kortum, Cancer Res. 63, 1999 (2003).

10. I. H. El-Sayed, X. Huang, and M. A. El-Sayed, Nano Lett. 5, 829 (2005).

11. J. J. Mock, S. J. Oldenburg, D. R. Smith, D. A. Schultz, and S. Schultz, Nano Lett. 2, 465 (2002).

12. S. R. Nicewarner-Pena, R. G. Freeman, B. D. Reiss, L. He, D. J. Pena, I. D. Walton, R. Cromer, C. D. Keating, and M. J. Natan, Science 2, 137 (2001).

13. P. P. Pompa, L. Martiradonna, A. Della Torre, F. Della Sala, L. Manna, M. De Vittorio, F. Calabi, R. Cingolani, and R. Rinaldi, Nature Nanotech. 1, 126 (2006).

14. J. W. Stone, P. N. Sisco, E. C. Goldsmith, S. C. Baxter, and C. J. Murphy, Nano Lett. 7, 116 (2007)

15. P. K. Jain, K. S. Lee, I. H. El-Sayed, and M. A. El-Sayed, Journ. Phys. Chem. B 110, 7238 (2006).

16. A. Rakic, A. B. Djurisic, J. M. Elazar, and M. L. Majewski, Appl. Opt. 37, 5271 (1998).

17. K. Kurihara and K. Suzuki, Anl. Chem. 74, 696 (2002).

18. M. Mitsushio, S. Higashi, and M. Higo, Anal. Sciences 19, 1421 (2003).

19. S. Franzen, J. Phys. Chem. C 112, 6027 (2008).

20. A. Kondilis, E. Aperathitis, and M. Modreanu, Thin solid Films 516, 8073 (2008). 
21. M. Chen and R. G. Horn, Journ. Coll. Interf. Science 315, 814 (2007).

22. Q. Zhang, J. J. Xu, Y. Liu, and H. Y. Cuen, Lab On Chip 8, 352 (2008).

23. C. E. Hoppe, C. Ridriguez-Abreu, M. Lazzari, M. A. LopezQuintela, and C. Solans, Phys. Stat. Sol. (a) 205, 1455 (2008).
24. K. L. Kelly, E. Coronado, L. L. Zhao, and G. C. Schatz, J. Phys. Chem. B 107, $668 \underline{\text { (2003)}}$.

25. A. Massaro, V. Errico, T. Stomeo, A. Salhi, R. Cingolani, A. Passaseo, and M. De Vittorio, IEEE Journ. Light. Technol. 26, 2960 (2008).

26. S. Caorsi, D. Moreno, and F. Sidoti, IEEE Trans. Antennas Propagat. 41, 1296 (1993).

Received: 28 July 2010. Accepted: 13 September 2010. 